\title{
ON THE EXISTENCE OF AN ABSOLUTELY MINIMAL NORM IN A BANACH ALGEBRA
}

\section{A. OLUBUMMO}

1. The norm $\|\cdot\|$ in a Banach algebra $A$ is said to be minimal [1] if, given any other norm $\|\cdot\|_{1}$ in $A$ (with respect to which $A$ need not be complete), the condition $\|a\|_{1} \leqq\|a\|$ for each $a \in A$ implies that $\|a\|_{1}$ $=\|a\|$. We shall say that $\|\cdot\|$ is absolutely minimal if, given any other norm $\|\cdot\|_{1}$ whatever in $A$, then $\|a\|_{1} \geqq\|a\|$ for each $a \in A$. An absolutely minimal norm is of course minimal.

Among the several examples of Banach algebras possessing a minimal norm, we mention (i) any complex $B^{*}$-algebra [1, Theorem 10], (ii) any left c.c. $B^{f}$-algebra $[3$, Theorem 3.2], (iii) any Banach algebra of operators on a Banach space $X$ which contains all operators in $X$ of finite rank with the uniform topology [ 1 Theorem 8]. The following example of a commutative Banach algebra with the stronger property of an absolutely minimal norm is due to Kaplansky [2, Theorem 6]:

TheOREM (KAPLANSKy). Let $C(X)$ be the commutative Banach algebra of all real or complex continuous functions vanishing at $\infty$ on a locally compact Hausdorff space $X$. Then the function norm in $C(X)$ is absolutely minimal.

In view of the obviously important role that an absolutely minimal norm would play in the structure theory of an abstract algebra possessing it, it is of some interest to know if any noncommutative Banach algebras have this property. In this note, we identify a fairly wide class of Banach algebras which do not possess an absolutely minimal norm: we show that a Banach algebra which can be regarded as an algebra of operators on a Banach space $X$, which contains all operators in $X$ of finite rank, cannot have an absolutely minimal norm unless in the trivial case in which $X$ is one-dimensional. This is done in $\S 2$ (Theorem 1). In $\S 3$, we give an example of a "slightly" noncommutative Banach algebra with an absolutely minimal norm: this is the algebra over the reals of bounded continuous quarternionvalued functions with a suitable norm.

2. The following lemma is well-known:

Lemma. Let $X$ be an arbitrary finite-dimensional Banach space. Then all norms in $X$ are equivalent; i.e. if $\|\cdot\|$ and $\|\cdot\|_{1}$ are any two norms in $X$, there exist $M, N$ such that $M\|x\| \leqq\|x\|_{1}$ and $N\|x\|_{1} \leqq\|x\|$ for $x \in X$.

Received by the editors October 12, 1959. 
The following is our main result.

Theorem 1. Let $A$ be an algebra of operators in a Banach space $X$ that contains all operators in $X$ of finite rank. If the dimension of $X$ is greater than one, then there does not exist any absolutely minimal norm in $A$.

Proof. Let $\|\cdot\|$ be the norm in $X$. Our first main step in the proof is to construct two norms $\|\cdot\|_{1},\|\cdot\|_{2}$ in $X$ and show that they are equivalent to $\|\cdot\|$.

Suppose that $U$ is a two-dimensional subspace of $X$; we construct a complement of $U$ as follows. Let $u_{1}, u_{2}$ be basis elements for $U$. Then there exist continuous linear functionals $\phi_{1}, \phi_{2}$ defined in $X$ such that $\phi_{1}\left(u_{1}\right)=0, \phi_{1}\left(u_{2}\right)=1$ and $\phi_{2}\left(u_{1}\right)=1, \phi_{2}\left(u_{2}\right)=0$. If $\phi_{i}^{\perp}$ $=\left[x \in X: \phi_{i}(x)=0\right]$, then the set $W=\phi_{1}^{\perp} \cap \phi_{2}^{\perp}$ is a complement of $U$. Thus every element $x \in X$ can be uniquely expressed as the sum of an element in $U$ and an element in $W$, and we write $X=U \oplus W$.

Let $x \in X$, with $x=u+w, u \in U, w \in W$. We first define a new norm $\|\cdot\|^{\prime}$ in $X$ by $\|x\|^{\prime}=\|u\|+\|w\|$. Then $\|\cdot\|^{\prime}$ is a proper norm and $\|x\| \leqq\|x\|^{\prime}$. Writing $u$ in the form $u=\phi_{1}(x) u_{1}+\phi_{2}(x) u_{2}$, we have

$$
\begin{aligned}
\|u\| & =\left\|\phi_{1}(x) u_{1}+\phi_{2}(x) u_{2}\right\| \leqq\left\|\phi_{1}(x) u_{1}\right\|+\left\|\phi_{2}(x) u_{2}\right\| \\
& =\left|\phi_{1}(x)\right|\left\|u_{1}\right\|+\left|\phi_{2}(x)\right|\left\|u_{2}\right\| \\
& \leqq\left\|\phi_{1}\right\| \cdot\|x\| \cdot\left\|u_{1}\right\|+\left\|\phi_{2}\right\|\|x\| \cdot\left\|u_{2}\right\| \\
& =K\|x\|,
\end{aligned}
$$

where $K=\left\|\phi_{1}\right\|\left\|u_{1}\right\|+\left\|\phi_{2}\right\|\left\|u_{2}\right\|$.

Now $w=x-u$ and $\|w\| \leqq\|x\|+\|u\| \leqq\|x\|+K\|x\| \leqq(1+K)\|x\|$. Then $\|x\|^{\prime} \leqq K\|x\|+(1+K)\|x\|=Q\|x\|$, where $Q=1+2 K$. The last inequality and the fact that $\|x\| \leqq\|x\|^{\prime}$ show that the norm $\|\cdot\|^{\prime}$ is equivalent to $\|\cdot\|$ and we may therefore set

$$
\|x\|=\|u\|+\|w\| \text {. }
$$

Suppose now that $V$ is the two-dimensional complex vector space of all ordered pairs $\left(\zeta_{1}, \zeta_{2}\right)$ of complex numbers. Since each $u \in U$ can be written as $u=\zeta_{1} u_{1}+\zeta_{2} u_{2}$, we may identify $U$ with $V$ by the correspondence $u \sim\left(\zeta_{1}, \zeta_{2}\right)$. We now define two norms $\left\|\cdot\left|\left\|\cdot\left|\left\|\cdot|\||^{\prime}\right.\right.\right.\right.\right.$ in $U$ by taking for $u \sim\left(\zeta_{1}, \zeta_{2}\right), \quad\|u\| \|=\left\{\left|\zeta_{1}\right|^{2}+\left|\zeta_{2}\right|^{2}\right\}^{1 / 2}$ and $\|u\|^{\prime}$ $=\max \left\{\left|\zeta_{1}\right|,\left|\zeta_{2}\right|\right\}$. With these two norms in $U$ we are able to define the two required norms $\|\cdot\|_{1},\|\cdot\|_{2}$ in $X$ by taking

$$
\|x\|_{1}=\|u\|\|+\| w \|
$$




$$
\|x\|_{2}=\|u\|\left\|^{\prime}+\right\| w \| \text {. }
$$

Comparing equations (ii) and (iii) with (i), and using Lemma 1, we have two new norms $\|\cdot\|_{1},\|\cdot\|_{2}$ in $X$ each of which is equivalent to $\|\cdot\|$. Thus $A$ contains all the operators of finite rank in $X$ that are bounded with respect to each of the norms $\|\cdot\|_{1}$ and $\|\cdot\|_{2}$.

Let $\|\cdot\|_{1},\|\cdot\|_{2}$ denote also the uniform norms in $A$ corresponding to $\|\cdot\|_{1}$ and $\|\cdot\|_{2}$ in $X$. We shall now show that if $|\cdot|$ is any norm in $A$ that is absolutely minimal, we have $\|\cdot\|_{1}=|\cdot|=\|\cdot\|_{2}$. In fact, we have $\|T\|_{1} \geqq|T|,\|T\|_{2} \geqq|T|$ for all $T \in A$. But since each of the norms $\|\cdot\|_{1},\|\cdot\|_{2}$ in $A$ is minimal being uniform norms in an algebra of operators in $X$, containing all operators of finite rank we must have $\|T\|_{1}=|T|=\|T\|_{2}$ for all $T \in A$.

We complete the proof by constructing a $\tilde{T} \in A$ with $\|\tilde{T}\|_{2}>\|\tilde{T}\|_{1}$. To do this we suppose that $T_{0}$ is a bounded linear transformation of $U$ into itself and consider the operator $\widetilde{T}$ defined by $\widetilde{T}(x)=\widetilde{T}(u+w)$ $=T_{0}(u)$. Then $\tilde{T} \in A$. Set $\left\|T_{0}\right\|_{U}=\sup _{\|\| u \| \mid \leq 1}\|\| T_{0}(u) \mid\|;\| T_{0} \|_{U}^{\prime}$ $=\sup _{\|\| u\|\|^{\prime} \leq 1} \mid\left\|T_{0}(u)\right\| \|$. Then $\|\widetilde{T}\|_{1}=\left\|T_{0}\right\|_{U}$ and $\|\widetilde{T}\|_{2}=\left\|T_{0}\right\|_{U}^{\prime}$. If $T_{0}$ is the operator given by the matrix

$$
T_{0}=\left(\begin{array}{cc}
1 & -1 / 2 \\
2 & -1
\end{array}\right)
$$

then this matrix sends $u \sim\left(\zeta_{1}, \zeta_{2}\right)$ into $T_{0}(u) \sim\left(\zeta_{1}-\zeta_{2} / 2,2 \zeta_{1}-\zeta_{2}\right)$. Thus

$$
\begin{aligned}
\left\|\tilde{T}_{1}\right\|=\sup _{\||u||| \leqq 1} \mid\left\|T_{0}(u)\right\| \| & =\sup _{\left|\zeta_{1}\right|^{2}+\left|\zeta_{2}\right|^{2} \leqq 1}\left[\left|\zeta_{1}-\zeta_{2} / 2\right|^{2}+\left|2 \zeta_{1}-\zeta_{2}\right|^{2}\right]^{1 / 2} \\
& =\sup _{\left|\zeta_{1}\right|^{2}+\left|\zeta_{2}\right|^{2} \leqq_{1}}\left(5^{1 / 2} / 2\right) \cdot\left|2 \zeta_{1}-\zeta_{2}\right|,
\end{aligned}
$$

and

$$
\begin{aligned}
\|\tilde{T}\|_{2}=\sup _{\|\left. u||\right|^{\prime} \leqq 1}\left\|T_{0}(u)\right\| \| & =\sup _{\max \left(\left|\zeta_{1}\right|,\left|\zeta_{2}\right|\right) \leqq 1}\left[\left|\zeta_{1}-\zeta_{2} / 2\right|,\left|2 \zeta_{1}-\zeta_{2}\right|\right] \\
& =\sup _{\max \left(\left|\zeta_{1}\right|,\left|\zeta_{2}\right|\right) \leqq 1}\left|2 \zeta_{1}-\zeta_{2}\right| .
\end{aligned}
$$

In order to calculate $\|\tilde{T}\|_{1}=\left\|T_{0}\right\|_{U}$, we consider the real twodimensional subspace $U_{0}$ of $U$. This corresponds to the subspace $V_{0}$ of $V$ where $V_{0}$ is the two-dimensional vector space over the real field $R$, of all ordered pairs $\left(\xi_{1}, \xi_{2}\right)$ of real numbers. Using the norm $\||\cdot|\| \mid$ in $U$, we show that the norm $\left\|T_{0}\right\|_{U_{0}}$ of $T_{0}$ as an operator in $U_{0}$ is the same as its norm $\left\|T_{0}\right\|_{U}$ as an operator in the whole of $U$. We have

$$
\left\|T_{0}\right\|_{U_{0}}=\sup _{\xi_{1}^{2}+\xi^{2} \leqq 1}\left\|T_{0}(u)\right\|, \quad u \sim\left(\xi_{1}, \xi_{2}\right) .
$$


It is clear that $\left\|T_{0}\right\|_{U_{0}} \leqq\left\|T_{0}\right\|_{v}$. On the other hand, for a complex vector $z=x+i y \in U$, we have

$$
\begin{aligned}
\left\|T _ { 0 } ( z ) \left|\left\|^{2}=\right\|\left\|T_{0}(x+i y) \mid\right\|^{2}\right.\right. & =\|\| T_{0}(x)+T_{0}(i y)\|\|^{2} \\
& =\|\| T_{0}(x)\|\|^{2}+\left\|T_{0}(y)\right\| \|^{2} \\
& \leqq\left\|T_{0}\right\|_{U_{0}}^{2}\left\{\|x \mid\|^{2}+\|\| y \|^{2}\right\} \\
& =\left\|T_{0}\right\|_{U_{0}}^{2}\|\|^{2} \|^{2} .
\end{aligned}
$$

Thus $\left|\left\|T_{0}(z)\right\|\|\leqq\| T_{0}\left\|_{U_{0}}\right\| z\right|\|, \quad\| T_{0}\left\|_{U} \leqq\right\| T_{0} \|_{U_{0}}$ and hence $\left\|T_{0}\right\|_{U_{0}}$ $=\left\|T_{0}\right\|_{U}$. We now calculate $\left\|T_{0}\right\|_{U_{0}}$.

$$
\left\|T_{0}\right\|_{U_{0}}=\sup _{\xi_{1}^{2}+\xi_{2}^{2} \leqq 1}\left(5^{1 / 2} / 2\right)\left|2 \xi_{1}-\xi_{2}\right|, \xi_{1}, \xi_{2} \in R .
$$

Write $\xi_{1}=\cos \theta, \xi_{2}=\sin \theta, 0 \leqq \theta \leqq 2 \pi$. Then

$$
\left\|T_{0}\right\|_{U_{0}}=\sup _{0 \leqq \theta \leqq 2 \pi}\left(5^{1 / 2} / 2\right)|2 \cos \theta-\sin \theta| \text {. }
$$

Now $2 \cos \theta-\sin \theta=5^{1 / 2}\left(2 / 5^{1 / 2} \cos \theta-1 / 5^{1 / 2} \sin \theta\right)=5^{1 / 2} \cos (\theta+\lambda)$, where $\lambda=\operatorname{arc} \cos \left(2 / 5^{1 / 2}\right)$. Therefore

$$
\sup _{0 \leqq \theta \leqq 2 \pi}|2 \cos \theta-\sin \theta|=5^{1 / 2},
$$

and we have $\|\tilde{T}\|_{1}=\left\|T_{0}\right\|_{U}=\left\|T_{0}\right\|_{U_{0}}=5 / 2$.

The supremum of $\left|2 \xi_{1}-\xi_{2}\right|$ for $\max \left(\left|\zeta_{1}\right|,\left|\zeta_{2}\right|\right) \leqq 1$ is attained at the point $(1,-1)$. This gives $\left\|T_{0}\right\|_{U}^{\prime}=\|\widetilde{T}\|_{2}=3$. Thus $\|\tilde{T}\|_{2}>\|\widetilde{T}\|_{1}$, which completes the proof.

3. Let $X$ be a topological space and $Q(x)$ the algebra over the real field of all bounded continuous quaternion-valued functions defined in $X$. Denoting the "norm" of a quaternion $q$ by $N(q)$, we define a norm $\|\cdot\|$ in $Q$ as follows:

If $x \in X$ and $f \in Q(X)$, we set $|f(x)|=\{N[f(x)]\}^{1 / 2}$, and take $\|f\|=\sup _{x \in X}|f(x)|$. Then $\|\cdot\|$ is a proper norm with respect to which $Q(x)$ is a Banach algebra. Also since $\left|f^{2}(x)\right|=|f(x)|^{2}$, we have $\left\|f^{2}\right\|$ $=\|f\|^{2}$. Moreover, if $\bar{q}$ is the "conjugate" of the quaternion $q$, we can define a *-operation in $Q(x)$ by taking $f^{*}(x)=[f(x)]-$ for $f \in Q(x)$. With this operation, $Q(x)$ becomes a real $B^{*}$-algebra.

We shall now show that the norm $\|\cdot\|$ in $Q(x)$ is absolutely minimal. We need the following result due to Rickart [4]:

TheOREM (RICKART). Let $A$ be a $B^{*}$-algebra which is algebraically embedded in an arbitrary Banach algebra $B$. If $x \in A$, then the spectral radii of $x$ in $A$ and in $B$ are always equal. 
Suppose that $\|\cdot\|_{1}$ is any new norm in $Q(x)$ and that $B$ is the completion of $Q(x)$ with respect to $\|\cdot\|_{1}$. Then by Rickart's result

$$
\lim _{n \rightarrow \infty}\left\|f^{n}\right\|^{1 / n}=\lim _{n \rightarrow \infty}\left\|f^{n}\right\|_{1}^{1 / n} \text { for each } f \in Q(x) .
$$

But since $\left\|f^{2}\right\|=\|f\|^{2}$ for each $f \in Q(x)$, we have

$$
\|f\|=\lim _{n \rightarrow \infty}\left\|f^{n}\right\|^{1 / n}=\lim _{n \rightarrow \infty}\left\|f^{n}\right\|_{1}^{1 / n} \leqq\|f\|_{1},
$$

and $\|\cdot\|$ is absolutely minimal.

\section{REFERENCES}

1. F. F. Bonsall, A minimal property of the norm in some Banach algebras, J. London Math. Soc. vol. 29 (1954) pp. 156-164.

2. I. Kaplansky, Normed algebras, Duke Math. J. vol. 16 (1949) pp. 399-417.

3. A. Olubummo, Left completely continuous $B^{f}$-algebras, J. London Math. Soc. vol. 32 (1957) pp. 270-276.

4. C. E. Rickart, On spectral permanence for certain Banach algebras, Proc. Amer. Math. Soc. vol. 4 (1953) pp. 191-196.

University Collega, IBADAN, NIGERIA 